\title{
Simple Parameters from Complete Blood Count Predict In-Hospital Mortality in COVID-19
}

Mattia Bellan, ${ }^{1,2}$ Danila Azzolina $\mathbb{D}^{1},{ }^{1}$ Eyal Hayden $\mathbb{D}^{1,2}$ Gianluca Gaidano ${ }^{1,2},{ }^{1,2}$

Mario Pirisi $\mathbb{D}^{1,2}$ Antonio Acquaviva $\mathbb{D}^{1,2}$ Gianluca Aimaretti $\mathbb{D}^{1,},{ }^{1,2}$ Paolo Aluffi Valletti $\mathbb{D}^{1,2}$ Roberto Angilletta $\mathbb{D}^{3},{ }^{3}$ Roberto Arioli $\mathbb{D}^{1,2}$ Gian Carlo Avanzi $\mathbb{D}^{1,2}$ Gianluca Avino $\mathbb{D}^{1,2}$ Piero Emilio Balbo $\mathbb{D}^{2}{ }^{2}$ Giulia Baldon $\mathbb{D}^{1}$, ${ }^{1,2}$ Francesca Baorda $\mathbb{D}^{1,4}$ Emanuela Barbero ${ }^{10}$, ${ }^{1,2}$ Alessio Baricich (D), ${ }^{1,2}$ Michela Barini ${ }^{D},{ }^{2}$ Francesco Barone-Adesi $\left(\mathbb{D},{ }^{1}\right.$ Sofia Battistini $(D),{ }^{1,2}$ Michela Beltrame $\mathbb{D}^{1,2}$ Matteo Bertoli ${ }^{1}{ }^{1,2}$, Stephanie Bertolin $\mathbb{D}^{1,2}$, Marinella Bertolotti ${ }^{1}{ }^{3}{ }^{3}$ Marta Betti $\left(\mathbb{D},{ }^{3}\right.$ Flavio Bobbio $\mathbb{D}^{2}{ }^{2}$ Paolo Boffano $\left(\mathbb{D},{ }^{1,2}\right.$ Lucio Boglione $\mathbb{D}^{1,4}$ Silvio Borrè ${ }^{1},{ }^{4}$ Matteo Brucoli $\mathbb{D}^{1,2}$ Elisa Calzaducca $\mathbb{D}^{1,},{ }^{1,2}$ Edoardo Cammarata $\mathbb{D}^{1,2}$ Vincenzo Cantaluppi $\mathbb{D}^{1,2}$ Roberto Cantello $\mathbb{D}^{1,2}$, Andrea Capponi ${ }^{1,2}{ }^{2}$ Alessandro Carriero $\left(\mathbb{D},{ }^{1,2}\right.$ Giuseppe Francesco Casciaro ${ }^{1 D},{ }^{1,2}$ Luigi Mario Castello $\left(\mathbb{D},{ }^{1,2}\right.$ Federico Ceruti $(\mathbb{D}),{ }^{1,2}$ Guido Chichino $\left(\mathbb{D},{ }^{3}\right.$ Emilio Chirico, ${ }^{1,2}$ Carlo Cisari $(\mathbb{D}),{ }^{1,2}$ Micol Giulia Cittone $\mathbb{D}^{1,2}$ Crizia Colombo ${ }^{10},{ }^{1,2}$ Cristoforo Comi ${ }^{1},{ }^{1,4}$ Eleonora Croce ${ }^{1},,^{1,4}$ Tommaso Daffara $\left(\mathbb{D},{ }^{1,2}\right.$ Pietro Danna $\mathbb{D}^{1,2}$ Francesco Della Corte ${ }^{1}{ }^{1,2}$

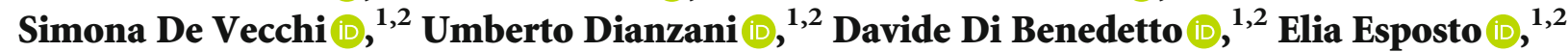
Fabrizio Faggiano $\left(\mathbb{D},{ }^{1}\right.$ Zeno Falaschi $\left(\mathbb{D},{ }^{1,2}\right.$ Daniela Ferrante $\left(\mathbb{D},{ }^{1}\right.$ Alice Ferrero $\left(\mathbb{D},{ }^{1,2}\right.$ Ileana Gagliardi $\mathbb{D}$, ${ }^{1,2}$ Alessandra Galbiati $\mathbb{D},,^{1,2}$ Silvia Gallo $\mathbb{D},,^{1,4}$ Pietro Luigi Garavelli $\mathbb{D}^{2}{ }^{2}$ Clara Ada Gardino $\left(\mathbb{D},{ }^{1,2}\right.$ Massimiliano Garzaro $\mathbb{D}^{1,2}$ Maria Luisa Gastaldello ${ }^{10},{ }^{1,2}$ Francesco Gavelli $\mathbb{D}^{1,2}$, Alessandra Gennari ${ }^{1,}{ }^{1,2}$ Greta Maria Giacomini ${ }^{10},{ }^{1,2}$ Irene Giacone $\mathbb{D}^{1,}{ }^{1,4}$ Valentina Giai Via $\mathbb{D}^{1,2}$ Francesca Giolitti ${ }^{10},{ }^{1,2}$ Laura Cristina Gironi $\mathbb{D}^{1,2}$ Carla Gramaglia $\mathbb{D}^{\mathbb{D}},,^{1,2}$ Leonardo Grisafi $\mathbb{D}^{1,},{ }^{1,2}$ Ilaria Inserra $\mathbb{D}^{1},{ }^{1,2}$ Marco Invernizzi $\mathbb{D}^{1},{ }^{1,2}$ Marco Krengli $(\mathbb{D}),{ }^{1,2}$ Emanuela Labella $\mathbb{D}^{1},{ }^{1,2}$ Irene Cecilia Landi $\left(\mathbb{D},{ }^{1,2}\right.$ Raffaella Landi $(\mathbb{D}),^{1,2}$ Ilaria Leone $(\mathbb{D})^{1,2}$ Veronica Lio ${ }^{(D)}{ }^{1,2}$ Luca Lorenzini ${ }^{1},,^{1,2}$

Antonio Maconi $\mathbb{D}^{3},{ }^{3}$ Mario Malerba $\mathbb{D}^{1},{ }^{1,4}$ Giulia Francesca Manfredi $\mathbb{D}$, ${ }^{1,2}$ Maria Martelli $\mathbb{D}^{1,2}$ Letizia Marzari $\mathbb{D}^{1,2}$ Paolo Marzullo $\mathbb{D}^{1,2}$ Marco Mennuni ${ }^{1,},{ }^{1,2}$

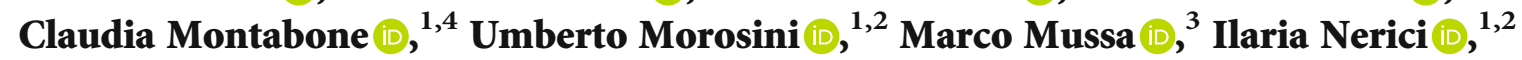
Alessandro Nuzzo (D), ${ }^{1,2}$ Carlo Olivieri (D), ${ }^{1,4}$ Samuel Alberto Padelli (iD, ${ }^{1,4}$ Massimiliano Panella $\left(\mathbb{D},{ }^{1}\right.$ Andrea Parisini $\left(\mathbb{D},{ }^{3}\right.$ Alessio Paschè $\left(\mathbb{D},{ }^{1,2}\right.$ Filippo Patrucco $\mathbb{D}$, ${ }^{1,2}$ Giuseppe Patti $\mathbb{D}^{1,2}$ Alberto Pau $\mathbb{D}^{1,2}$ Anita Rebecca Pedrinelli $\mathbb{D},{ }^{1,2}$ Ilaria Percivale $\mathbb{D}$, ${ }^{1,2}$ Luca Ragazzoni $\mathbb{D}^{1},{ }^{1}$ Roberta $\operatorname{Re} \mathbb{D}^{1},{ }^{1,4}$ Cristina Rigamonti ${ }^{1},{ }^{1,2}$ Eleonora Rizzi $\mathbb{D}^{1,2}$ Andrea Rognoni $\mathbb{D}^{1,2}$ Annalisa Roveta ${ }^{1},{ }^{3}$ Luigia Salamina ${ }^{1},{ }^{2}$ Matteo Santagostino ${ }^{1}$, ${ }^{1,2}$ Massimo Saraceno $\mathbb{D}^{1,2}$ Paola Savoia $\mathbb{D}^{1,2}$ Marco Sciarra $\mathbb{D}^{1,3}$ Andrea Schimmenti $\mathbb{D}^{3}{ }^{3}$

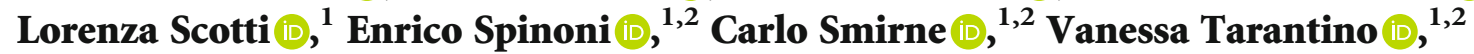
Paolo Amedeo Tillio $\mathbb{D}^{1,4}$ Stelvio Tonello ${ }^{1},{ }^{1}$ Rosanna Vaschetto $\mathbb{D}^{1,2}$ Veronica Vassia $\mathbb{D}^{1,},{ }^{1,2}$ Domenico Zagaria (D), ${ }^{1,2}$ Elisa Zavattaro (D), ${ }^{1,2}$ Patrizia Zeppegno ${ }^{1}$, ${ }^{1,2}$

Francesca Zottarelli $\mathbb{D}^{1,2}$ and Pier Paolo Sainaghi ${ }^{1,2}$ 


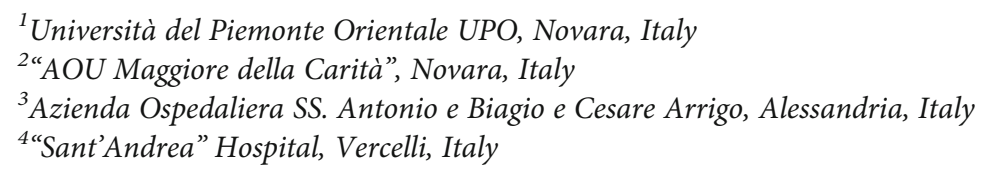

Correspondence should be addressed to Pier Paolo Sainaghi; pierpaolo.sainaghi@med.uniupo.it

Received 12 July 2020; Revised 9 November 2020; Accepted 22 December 2020; Published 13 May 2021

Academic Editor: Wen-Jun Tu

Copyright ( 2021 Mattia Bellan et al. This is an open access article distributed under the Creative Commons Attribution License, which permits unrestricted use, distribution, and reproduction in any medium, provided the original work is properly cited.

Introduction. The clinical course of Coronavirus Disease 2019 (COVID-19) is highly heterogenous, ranging from asymptomatic to fatal forms. The identification of clinical and laboratory predictors of poor prognosis may assist clinicians in monitoring strategies and therapeutic decisions. Materials and Methods. In this study, we retrospectively assessed the prognostic value of a simple tool, the complete blood count, on a cohort of 664 patients ( $F$ 260; 39\%, median age 70 (56-81) years) hospitalized for COVID-19 in Northern Italy. We collected demographic data along with complete blood cell count; moreover, the outcome of the hospital in-stay was recorded. Results. At data cut-off, 221/664 patients (33.3\%) had died and 453/664 (66.7\%) had been discharged. Red cell distribution width (RDW) $\left(\chi^{2} 10.4 ; p<0.001\right)$, neutrophil-to-lymphocyte (NL) ratio $\left(\chi^{2} 7.6 ; p=0.006\right)$, and platelet count $\left(\chi^{2} 5.39 ; p=0.02\right)$, along with age $\left(\chi^{2} 87.6 ; p<0.001\right)$ and gender $\left(\chi^{2} 17.3 ; p<0.001\right)$, accurately predicted in-hospital mortality. Hemoglobin levels were not associated with mortality. We also identified the best cut-off for mortality prediction: a NL ratio $>4.68$ was characterized by an odds ratio for in-hospital mortality $(\mathrm{OR})=3.40(2.40-4.82)$, while the OR for a RDW $>13.7 \%$ was 4.09 (2.87-5.83); a platelet count $>166,000 / \mu \mathrm{L}$ was, conversely, protective (OR: $0.45(0.32-0.63)$ ). Conclusion. Our findings arise the opportunity of stratifying COVID-19 severity according to simple lab parameters, which may drive clinical decisions about monitoring and treatment.

\section{Introduction}

The global spread of severe acute respiratory syndrome coronavirus 2 (SARS-CoV-2) generated a pandemic outbreak in late 2019-early 2020, which severely impacted on care delivery worldwide. The clinical picture caused by the abovementioned viral infection has been called Coronavirus disease 2019 (COVID-19): its clinical manifestations encompass a wide range of entities, from a mild flu-like illness to life-threatening forms [1]. Despite differences among countries, the overall in-hospital mortality is high, being reported up to $30 \%$ [2]. The reasons underlying such a different clinical behavior are still unknown; thus, it is difficult to predict who will develop a severe clinical picture and who, conversely, will show a mild disease.

Thus, the identification of predictors of mortality might be valuable to adjust clinicians' approach to monitoring and therapy. Among clinical parameters, proposed predictors of poor prognosis include age, comorbidities, and male gender [3,4]. Moreover, some laboratory variables have been associated to mortality in different cohorts $[5,6]$; in a recent meta-analysis, elevated cardiac troponin, C-reactive protein, interleukin-6, D-dimer, creatinine, liver function tests and decreased levels of albumin turned out to predict mortality among COVID-19 hospitalized patients [7]. Furthermore, preliminary reports suggest that blood cell count may be particularly relevant $[8,9]$. Blood cell count is a simple, highly informative, and universally available exam. The validation of these early reports may give to clinicians a further valuable tool for clinical risk stratification. On these grounds, here we aimed to verify whether simple data retrievable from blood cell count may predict the out- come in patients admitted to the hospital because of COVID-19.

\section{Methods}

2.1. Study Population. The study was conducted at the "Maggiore della Carità" University Hospital in Novara, Northern Italy.

Scanning the hospital administrative database, we retrieved all consecutive patients older than 18 years of age, admitted to the hospital after emergency room evaluation, with a confirmed diagnosis of SARS-CoV-2 infection by reverse-transcriptase polymerase chain reaction (RT-PCR) of a nasopharyngeal swab, between $1^{\text {st }}$ March 2020 and $28^{\text {th }}$ April 2020. We identified a total of 763 patients.

We revised data available from central lab software to retrieve the complete blood cell count at hospital admission, along with gender and age. We also recorded the outcome of in-hospital stay (discharged or deceased).

The study protocol was approved by the Institutional Review Board (Comitato Etico Interaziendale Novara; IRB code CE 97/20) and conducted in strict accordance with the principles of the Declaration of Helsinki. Prospective informed consent was waived by competent authorities due to the retrospective nature of the study and the use of pseudonymized data.

2.2. Statistical Analysis. Data were summarized according to groups as median and (25th-75th percentile) and analyzed using the Wilcoxon test. Categorical variables, whenever dichotomous or nominal, were reported as frequencies and percentages and analyzed through the Chi-square test. 
TABLE 1: Clinical features of the study population. The main general features of the study population are reported. Categorical variables, whenever dichotomous or nominal, were reported as frequencies and percentages and analyzed through the Chi-square test.

\begin{tabular}{|c|c|c|c|c|}
\hline & Total (N 664) & Discharged (N 453) & Dead (N 211) & $p$ \\
\hline Age (years) & $70(56-81)$ & $63(52-75)$ & $80(73-87)$ & $<0.0001$ \\
\hline Gender (M/F) & $404(61) / 260(39)$ & $260(57) / 193(43)$ & $144(68) / 67(32)$ & 0.008 \\
\hline $\mathrm{WBC}\left(\times 10^{3} / \mu \mathrm{L}\right)$ & $7.3(5.2-10.9)$ & $6.8(5.1-9.7)$ & $8.4(5.8-12.0)$ & $<0.0001$ \\
\hline Neutrophils $\left(\times 10^{3} / \mu \mathrm{L}\right)$ & $5.3(3.5-8.6)$ & $4.8(3.4-7.6)$ & $6.7(4.1-9.6)$ & $<0.0001$ \\
\hline Lymphocytes $\left(\times 10^{3} / \mu \mathrm{L}\right)$ & $1.07(0.76-1.54)$ & $1.15(0.82-1.56)$ & $0.92(0.66-1.92)$ & $<0.0001$ \\
\hline Eosinophils $\left(\times 10^{3} / \mu \mathrm{L}\right)$ & $0.01(0.00-0.03)$ & $0.01(0.00-0.04)$ & $0.00(0.00-0.01)$ & $<0.0001$ \\
\hline NL ratio & $4.7(2.8-9.1)$ & $4.0(2.5-7.0)$ & $7.0(3.8-13.2)$ & $<0.0001$ \\
\hline Platelets $\left(\times 10^{3} / \mu \mathrm{L}\right)$ & $195(149-249)$ & $198(157-250)$ & $168(129-243)$ & $<0.0001$ \\
\hline $\mathrm{Hb}(\mathrm{gr} / \mathrm{dL})$ & $13.4(11.8-14.7)$ & $13.7(12.1-14.8)$ & $12.9(11.4-14.4)$ & 0.08 \\
\hline $\mathrm{MCH}(\mathrm{pg})$ & $29.5(28.2-30.7)$ & $29.3(28.3-30.5)$ & $29.8(28.0-31.1)$ & 0.04 \\
\hline MCV (fL) & $89.5(85.7-93.4)$ & $88.8(85.1-91.9)$ & $91.3(86.9-94.6)$ & $<0.0001$ \\
\hline RDW (\%) & $13.7(12.9-15.1)$ & $13.3(12.7-14.4)$ & $14.5(13.5-15.9)$ & $<0.0001$ \\
\hline
\end{tabular}

Comparison between survivors and dead has been performed by a nonparametric Wilcoxon test. For abbreviation: M: males; F: females; WBC: white blood cells; NL ratio: neutrophil-to-lymphocyte ratio; MCH: mean cell hemoglobin; MCV: mean corpuscular volume; RDW: red cell distribution width.

The optimal cut-off value for each predictor has been computed considering as criterion the sum of sensitivity and specificity maximization [10]. The cut-off values have been considered to dichotomize the predictors; the area under curve (AUC) values in discriminating the in-hospital mortality have been reported.

Univariate analysis and multivariate logistic regression were carried out to quantify the effects of the dichotomized covariates on in-hospital mortality. We included in the multivariate models the following covariates: age, gender, and laboratory parameters derived from complete blood count.

The .632 bootstrap (1000 resamples) validation procedure was carried out to evaluate the predictive logistic regression model performance reporting the Harrell-C statistics corrected for overoptimism [11].

The $p$ values have been adjusted via Holm multiple testing procedure.

Statistical analyses were conducted using R 3.5.2 with the rms packages [12]. The threshold of statistical significance was 0.05 for all tests used (two tailed).

\section{Results}

At data cut-off, 664 (260 females, 39\%; median age 70 (56-81) years) patients completed their hospital in-stay: 221/664 patients (33.3\%) had died and 453/664 (66.7\%) had been discharged. The median time to death was 6 days [2-10], while the median time to discharge was 8 days [5-14] $(p<0.001)$.

Table 1 reports the main features of the study population. The proportion of males was higher among those who died in-hospital; moreover, the age was significantly higher in this group of subjects, who also showed increased white blood cells (WBC), absolute neutrophil count (ANC), neutrophilto-lymphocyte ratio (NL ratio), eosinophil count, mean corpuscular hemoglobin $(\mathrm{MCH})$, mean corpuscular volume $(\mathrm{MCV})$, and red cell distribution width (RDW). Conversely, survivors had significantly higher platelet count, hemoglobin concentration, and absolute lymphocyte count (ALC).
TABLE 2: Multivariable model. The table shows a multivariable model including all blood count-derived variables.

\begin{tabular}{lcc}
\hline & $\chi^{2}$ & $p$ \\
\hline Age (years) & 77.04 & $<0.0001$ \\
Gender $(\mathrm{M} / \mathrm{F})$ & 17.3 & $<0.0001$ \\
WBC $\left(\times 10^{3} / \mu \mathrm{L}\right)$ & 3.63 & 0.06 \\
Neutrophils $\left(\times 10^{3} / \mu \mathrm{L}\right)$ & 4.25 & 0.04 \\
Lymphocytes $\left(\times 10^{3} / \mu \mathrm{L}\right)$ & 3.78 & 0.05 \\
Eosinophils $\left(\times 10^{3} / \mu \mathrm{L}\right)$ & 1.71 & 0.19 \\
NL ratio & 0.48 & 0.49 \\
Platelets $\left(\times 10^{3} / \mu \mathrm{L}\right)$ & 3.74 & 0.05 \\
Hb (gr/dL) & 0.37 & 0.54 \\
MCH (pg) & 0.56 & 0.45 \\
MCV (fL) & 0.32 & 0.57 \\
RDW (\%) & 12.74 & $<0.0001$ \\
\hline
\end{tabular}

For abbreviation: M: males; F: females; WBC: white blood cells; NL ratio: neutrophil-to-lymphocyte ratio; MCH: mean cell hemoglobin; MCV: mean corpuscular volume; RDW: red cell distribution width.

We ran a first multivariate model to test the predictive role of all the parameters derived from complete blood count. The results are shown in Table 2. Along with age and gender, RDW was the strongest independent predictor of mortality; ANC was still significantly and independently predictive of in-hospital mortality, while the contribution given by WBC, ALC, and platelet count was weaker. Predictably, the effect of NL ratio was blunted by the inclusion of ANC and ALC into the model.

On the basis of the first model, we ran a further multivariate analysis, including age, gender, platelet count, NL ratio (used as a summary measure for white blood cell count and differential), RDW, and hemoglobin. RDW ( $\chi^{2} 10.40$; $p<0.001)$, NL ratio $\left(\chi^{2} 7.59 ; p=0.006\right)$, and platelet count $\left(\chi^{2}\right.$ 5.39; $\left.p=0.02\right)$, along with age $\left(\chi^{2} 87.6 ; p<0.001\right)$ and gender $\left(\chi^{2}\right.$ 17.3; $p<0.001$, confirmed their independent 
TABLE 3: Univariable analysis. The optimal cut-off value for each predictor has been reported together with AUC (area under the curve) values. The univariable logistic regression OR (odds ratio) together with $95 \%$ confidence interval (CI) have been also reported.

\begin{tabular}{lcccc}
\hline & Cut-off & AUC & OR $($ CI95\%) & $p$ \\
\hline Age $($ years $)$ & 74 & 0.786 & $6.90(4.80-9.93)$ & $1.98(1.42-2.77)$ \\
WBC $\left(\times 10^{3} / \mu \mathrm{L}\right)$ & 8,360 & 0.587 & $2.56(1.83-3.59)$ & $<0.0001$ \\
Neutrophils $\left(\times 10^{3} / \mu \mathrm{L}\right)$ & 6,310 & 0.617 & $0.42(0.30-0.60)$ & $<0.0001$ \\
Lymphocytes $\left(\times 10^{3} / \mu \mathrm{L}\right)$ & 1,130 & 0.618 & $0.57(0.41-0.80)$ & $<0.0001$ \\
Eosinophils $\left(\times 10^{3} / \mu \mathrm{L}\right)$ & 0,100 & 0.587 & $3.40(2.40-4.82)$ & 0.0001 \\
NL ratio & 4.68 & 0.661 & $0.45(0.32-0.63)$ & $<0.0001$ \\
Platelets $\left(\times 10^{3} / \mu \mathrm{L}\right)$ & 166 & 0.582 & $0.60(0.39-0.75)$ & 0.00001 \\
Hb $(\mathrm{gr} / \mathrm{dL})$ & 13.0 & 0.577 & $1.84(1.26-2.68)$ & 0.0001 \\
MCH $(\mathrm{pg})$ & 30.8 & 0.549 & $2.51(1.80-3.52)$ & $<0.0001$ \\
MCV $(\mathrm{fL})$ & 90.6 & 0.602 & 0.689 & $(2.87-5.83)$ \\
RDW $(\%)$ & 13.7 & & $<0.0001$ \\
\hline
\end{tabular}

predictive role. Conversely, hemoglobin $\left(\chi^{2} 2.28 ; p=0.131\right)$ was not associated with outcome.

We further tried to identify the best performing cut-off for these parameters; in Table 3, we reported our results, with the respective area under the curve (AUC) and odds ratio.

On these bases, we built a final multivariate model which confirmed that age $>74$ years $\left(\chi^{2} 67.1 ; p<0.001\right)$, male gen$\operatorname{der}\left(\chi^{2} 14.9 ; p<0.001\right)$, platelets $<166,000\left(\chi^{2} 22.3 ; p<\right.$ $0.0001)$, NL ratio $>4.68\left(\chi^{2} 21.4 ; p<0.0001\right)$, and RDW $>$ $13.7 \%\left(\chi^{2} 25.2 ; p<0.001\right)$ were independent predictors of in-hospital mortality. Once again, hemoglobin $\left(\chi^{2} 0.03 ; p=\right.$ 0.85 ) was not associated to in-hospital mortality.

\section{Discussion}

In the present paper, we investigated a cohort of hospitalized patients in Northern Italy with a defined diagnosis of COVID-19, showing that simple complete blood count parameters obtained at hospital admission accurately predict in-hospital mortality. A first finding deserving a comment is the observed mortality which, in our cohort, was very high. More than $30 \%$ of patients died during hospital stay. This is in line with previous observations from other groups [13-15], although data about in-hospital mortality widely vary, with case fatality rates much lower in the first reported cohorts from China $[16,17]$. The reasons of this discrepancy are still largely unknown; however, both the phase of the epidemic curve and the geographic region have an impact in determining a very different prognosis in COVID-19 patients. Our study is adequately powered to compensate for variations in the mortality rate: for example, with a NL ratio above 7 in $25 \%$ of discharged patients and $50 \%$ of deceased patients, the estimated power is 0.9999 for at a mortality rate of $0.33(33 \%)$ and remains $>0.80$ for all populations with mortality rate above $0.06(6 \%)$. Thus, our data strongly support the idea that complete blood count, a routine test for most patients admitted to hospital, might be highly informative with regard to prognosis of COVID-19 patients admitted to hospital. This is in line with different studies reporting a potential role for blood count in the prediction of mortality in the context of Emergency Medicine, cardiovascular and cerebrovascular diseases [18-21].

More specifically, we identified three main predictors of in-hospital death: NL ratio, platelet count, and RDW.

NL ratio has already been described to be strongly associated with COVID-19 prognosis [22-24]. The ratio magnifies the strength of the association of both components, neutrophil count and lymphocyte count, in predicting the outcome for COVID-19 patients. Whether neutrophilia is just a biomarker of disease severity or has a pathogenetic role is unclear. However it is worth mentioning that COVID-19 mortality seems to be frequently related to the development of thromboembolic complications and systemic inflammation $[25,26]$. Neutrophilia is a known marker of venous thrombosis, possibly contributing by driving a necroinflammatory response [27]. Furthermore, neutrophils play their protective role not only through phagocytosis but also exploiting neutrophil extracellular trap (NET) formation [28]. Although NETs are beneficial in the host defense against pathogens, sustained NET formation may drive collateral damage in different human conditions, including viral infections [29]. It has been postulated that, in COVID19 , the inappropriate NET production might be crucial in the development of the "cytokine storm" responsible of acute respiratory distress syndrome (ARDS), severe inflammatory response syndrome (SIRS), and sepsis [27]. With respect to lymphocytes, it has been suggested that SARS-CoV-2 infection may primarily affect $\mathrm{T}$ lymphocytes [30] and that lymphopenia in severe patients is mainly related to a decreased ALC, especially CD8+ T cells [31]. Since T cells are important for dampening overactive innate immune responses during viral infection, their loss during SARS-CoV-2 infection may result in a more severe inflammatory responses [32].

We suggest to use NL ratio, in this context, being its prognostic value higher, as shown by our data. In fact, NL ratio magnifies the predictive potential of neutrophilia and lymphopenia, being 4.68 the best prognostic cut-off.

With respect to platelets, some authors suggested that thrombocytopenia may be a negative prognostic factor. In a recent meta-analysis on 31 studies including 7163 patients, a lower platelet count predicted severe COVID-19 [33]; the 
mechanism by which SARS-CoV-2 leads to platelet consumption, especially in case of severe disease, is unknown. A possible explanation is that the lung damage might lead to platelet activation and aggregation, finally causing thrombocytopenia [34]. A limitation of our study is that we did not exclude patients affected by liver cirrhosis, which is tipically associated to thrombocytopenia; this might have partially biased our finding [35].

Finally, we demonstrated that RDW is a very strong predictor of mortality. According to our data, RDW is characterized by the highest $\chi^{2}$ and OR for in-hospital mortality among all the variables considered. The clinical value of RDW is often neglected; in the last few years, it has been recognized as a common prognostic predictor in many human diseases: acute kidney injury, cancer, acute pancreatitis, and respiratory failure [36-40]. The predictive significance of RDW in the context of COVID-19 might reflect the effect on erythropoiesis of respiratory failure and systemic inflammation [41, 42].

Our study has some limitations, due to the context in which we were forced to act by the pandemic; in particular, the observational nature of the study limited data availability and did not allow us to correct for potential confounders. Despite these limitations, our findings may raise the opportunity of stratifying disease severity according to simple lab parameters, which may drive clinical decisions about monitoring and treatment.

\section{Conclusions}

In the present study, we demonstrated that some simple parameters derived from complete blood cell count have prognostic implications in the course of COVID-19, being able to identify those patients at higher risk of in-hospital mortality. The confirmation of these preliminary observations may make available to clinicians novel useful tools for prognostic stratification.

\section{Data Availability}

Data are available upon reasonable request to the corresponding author.

\section{Conflicts of Interest}

The authors have no conflict of interest to declare.

\section{Authors' Contributions}

PPS had full access to all of the data in the study and takes responsibility for the integrity of the data and the accuracy of the data analysis. MB, MP, GCA, and PPS conceptualized and designed the study. AA, GA, PAV, RA, RA, GA, PEB, $\mathrm{GB}, \mathrm{FB}, \mathrm{EB}, \mathrm{AB}, \mathrm{MB}, \mathrm{SB}, \mathrm{MB}, \mathrm{MB}, \mathrm{SB}, \mathrm{MB}, \mathrm{MB}, \mathrm{FB}, \mathrm{PB}$, $\mathrm{LB}, \mathrm{SB}, \mathrm{MB}, \mathrm{EC}, \mathrm{EC}, \mathrm{VC}, \mathrm{RC}, \mathrm{AC}, \mathrm{AC}, \mathrm{GFC}, \mathrm{LMC}, \mathrm{FC}, \mathrm{GC}$, EC, CC, MGC, CC, CC, EC, TD, PD, FDC, SDV, UD, DDB, EE, ZF, DF, AF, IG, GG, AG, SG, PLG, CAG, MG, MLG, FG, AG, GMG, IG, VGV, FG, LCG, CG, LG, II, MI, MK, EL, ICL, RL, IL, VL, LL, AM, MM, GFM, MM, LM, PM, MM, CM, UM, MM, IN, AN, CO, SAP, AP, AP, FP,
GP, AP, ARP, IP, LR, RR, CR, ER, AR, AR, LS, MS, MS, PS, MS, AS, LS, ES, CS, VT, PAT, ST, RV, VV, DZ, EZ, PZ, and FZ acquired the data. MB, DA, EH, GG, FBA, FF, MP, and PPS analyzed and interpreted the data. MB made the draft of the manuscript. All the authors critically revised the manuscript for important intellectual content. DA, EH, and PPS did the statistical analysis. EH, AC, and LS did the administrative, technical, or material support. GP, MP, GCA, and PPS supervised study.

\section{Acknowledgments}

We would like to thank for their massive contribution to clinical data collection all the COVID UPO Clinical Team: Giuseppe Aiosa, ${ }^{4}$ Andrea Airoldi, ${ }^{2}$ Ambra Barco, ${ }^{2}$ Olivia Bargiacchi, ${ }^{2}$ Simona Bazzano, ${ }^{2}$ Paola Berni, ${ }^{2}$ Bianca Bianchi, ${ }^{2}$ Sara Bianco, ${ }^{2}$ Stefano Biffi, ${ }^{2}$ Valeria Binda, ${ }^{2}$ Tatiana Bolgeo, ${ }^{4}$ Cesare Bolla, ${ }^{4}$ Valeria Bonato, ${ }^{4}$ Giacomo Bonizzoni, ${ }^{4}$ Alice Bragantini, ${ }^{4}$ Diego Brustia, ${ }^{2}$ Valentina Bullara, ${ }^{2}$ Michela Burlone, ${ }^{2}$ Fabio Brustia, ${ }^{2}$ Stefano Caccia, ${ }^{2}$ Anna Calareso, ${ }^{3}$ Gianmaria Cammarota, ${ }^{2}$ Laura Cancelliere, ${ }^{2}$ Roberto Carbone, ${ }^{4}$ Antonella Cassinari, ${ }^{4}$ Elisa Ceriani, ${ }^{2}$ Tiziana Cena, ${ }^{2}$ Elisa Clivati, ${ }^{2}$ Laura Collimedaglia ${ }^{3}$, Andrea Colombatto, ${ }^{2}$ Cristina Cornella, ${ }^{2}$ Martina Costanzo, ${ }^{1,2}$ Alessandro Croce, ${ }^{1,2}$ Carla De Benedittis, ${ }^{1,2}$ Stefania Delorenzi, ${ }^{4}$ Rosa Dionisio, ${ }^{4}$ Paolo Donato, ${ }^{2}$ Maria Esposito, ${ }^{2}$ Stefano Fangazio, ${ }^{2}$ Alessandro Feggi, ${ }^{2}$ Sara Ferrillo, ${ }^{4}$ Valentina Foci, ${ }^{3}$ Gian Paolo Fra, ${ }^{2}$ Claudio Gaggino, ${ }^{4}$ Eleonora Gambaro, ${ }^{2}$ Eleonora Gattoni, ${ }^{2}$ Luca Gattoni, ${ }^{2}$ Fabio Giacchero, ${ }^{4}$ Romina Gianfreda, ${ }^{4}$ Ailia Giubertoni, ${ }^{2}$ Lorenzo Grecu, ${ }^{4}$ Francesca Grossi, ${ }^{2}$ Gabriele Guglielmetti, ${ }^{2}$ Stefania Guido, ${ }^{2}$ Giacomo Iannantuoni, ${ }^{2}$ Susanna Ingrao, ${ }^{2}$ Amalia Jona, ${ }^{2}{ }^{2}$ Elisa Lazzarich, ${ }^{2}$ Raffaella Lissandrin, ${ }^{4}$ Elisabetta Maduli, ${ }^{2}$ Federica Magnè, ${ }^{4}$ Eugenio Mantia, ${ }^{4}$ Debora Marangon, ${ }^{2}$ Maurilio Massara, ${ }^{2}$ Erica Matino, ${ }^{2}$ Maria Grazia Mauri, ${ }^{2}$ Mirta Menegatti, ${ }^{2}$ Roberta Moglia, ${ }^{2}$ Rossella Molinari, ${ }^{2}$ Stefania Morelli, ${ }^{2}$ Paola Morlino, ${ }^{2}$ Paola Naldi, ${ }^{2}$ Claudio Nebbiolo, ${ }^{2}$ Pinuccia Omodeo, ${ }^{4}$ Daniela Palmieri, ${ }^{2}$ Antonio Panero, ${ }^{2}$ Massimiliano Parodi, ${ }^{4}$ Roberta Pedrazzoli, ${ }^{2}$ Carolina Pelazza, ${ }^{4}$ Serena Penpa, ${ }^{4}$ Raffaella Perucca, ${ }^{2}$ Alice Pirovano, ${ }^{1,2}$ Sergio Pittau, ${ }^{2}$ Patrizia Pochetti, ${ }^{3}$ Federica Poletti, ${ }^{2}$ Biagio Polla, ${ }^{4}$ Paolo Prandi, ${ }^{2}$ Flavia Prodam, ${ }^{1,2}$ Pierluigi Prosperini, ${ }^{2}$ Alessia Puma, ${ }^{2}$ Marco Quaglia, Alberto Raie, ${ }^{3}$ Rachele Rapetti, ${ }^{2}$ Silvia Ravera, ${ }^{4}$ Azzurra Re, ${ }^{2}$ Matia Reale, ${ }^{4}$ Antonella Rossati, ${ }^{2}$ Maura Rossi, ${ }^{4}$ Paola Rossi, ${ }^{4}$ Roberto Rostagno, ${ }^{2}$ Annalisa Roveta, $\mathrm{MD}^{4}$ Giulia Salomoni, ${ }^{4}$ Maria Teresa Samà, ${ }^{2}$ Eleonora Sarchi, ${ }^{4}$ Maddalena Sarcoli, ${ }^{2}$ Cristina Sarda, ${ }^{4}$ Ilaria Sguazzotti, ${ }^{2}$ Daniele Soddu, ${ }^{1,2}$ Daniele Sola, ${ }^{2}$ Paolo Stobbione, ${ }^{4}$ Monica Todoerti, ${ }^{4}$ Gian Carlo Vallese, ${ }^{2}$ Claudia Varrasi, ${ }^{2}$ Alessia Veia, ${ }^{2}$ Gian Luca Vignazia, ${ }^{2}$ Isabella Zanotti, ${ }^{2}$ Erika Zecca, ${ }^{1,2}$ Daniela Zichittella, Giuliana Zisa, ${ }^{2}$ Elisabetta Zoppis. ${ }^{2}$

\section{References}

[1] N. Chen, M. Zhou, X. Dong et al., "Epidemiological and clinical characteristics of 99 cases of 2019 novel coronavirus pneumonia in Wuhan, China: a descriptive study," The Lancet, vol. 395 , no. 10223 , pp. 507-513, 2020. 
[2] A. B. Docherty, E. M. Harrison, C. A. Green et al., "Features of 20133 UK patients in hospital with covid-19 using the ISARIC WHO clinical characterisation protocol: prospective observational cohort study," BMJ, vol. 369, article m1985, 2020.

[3] J. B. Galloway, S. Norton, R. D. Barker et al., "A clinical risk score to identify patients with COVID-19 at high risk of critical care admission or death: an observational cohort study," The Journal of Infection, vol. 81, pp. 282-288, 2020.

[4] Z. Imam, F. Odish, I. Gill et al., "Older age and comorbidity are independent mortality predictors in a large cohort of 1305 COVID-19 patients in Michigan, United States," Journal of Internal Medicine, vol. 288, no. 4, pp. 469-476, 2020.

[5] S. Piano, A. Dalbeni, E. Vettore et al., "Abnormal liver function tests predict transfer to intensive care unit and death in COVID19," Liver International, vol. 40, no. 10, pp. 2394-2406, 2020.

[6] R. Herlekar, A. S. Roy, and M. Matson, "Hypoalbuminaemia in COVID-19 infection: a predictor of severity or a potential therapeutic target?," Journal of Medical Virology, vol. 93, no. 1, pp. 83-84, 2020.

[7] W. Tian, W. Jiang, J. Yao et al., "Predictors of mortality in hospitalized COVID-19 patients: a systematic review and metaanalysis," Journal of Medical Virology, vol. 92, no. 10, pp. 1875-1883, 2020.

[8] H. Sun, R. Ning, Y. Tao et al., "Risk factors for mortality in 244 older adults with COVID-19 in Wuhan, China: a retrospective study," Journal of the American Geriatrics Society, vol. 68, no. 6, pp. E19-E23, 2020.

[9] X. Yan, F. Li, X. Wang et al., "Neutrophil to lymphocyte ratio as prognostic and predictive factor in patients with coronavirus disease 2019: a retrospective cross-sectional study," Journal of Medical Virology, vol. 92, no. 11, pp. 2573-2581, 2020.

[10] X. Robin, N. Turck, A. Hainard et al., "pROC: an open-source package for R and S+ to analyze and compare ROC curves," BMC Bioinformatics, vol. 12, no. 1, p. 77, 2011.

[11] H. FEJ, "rms: regression modeling strategies. R package version 4.1-3," 2014, http://CRAN.R-project.org/package=rms.

[12] R Core Team, R: A Language and Environment for Statistical Computing, R Foundation for Statistical Computing, Vienna, Austria, 2019, https://www.R-project.org.

[13] F. Zhou, T. Yu, R. Du et al., "Clinical course and risk factors for mortality of adult inpatients with COVID-19 in Wuhan, China: a retrospective cohort study," The Lancet, vol. 395, no. 10229, p. 1038, 2020.

[14] G. Grasselli, A. Zangrillo, A. Zanella et al., "Baseline characteristics and outcomes of 1591 patients infected with SARS-CoV2 admitted to ICUs of the Lombardy region, Italy," JAMA, vol. 323, no. 16, pp. 1574-1581, 2020.

[15] S. Richardson, J. S. Hirsch, M. Narasimhan et al., "Presenting characteristics, comorbidities, and outcomes among 5700 patients hospitalized with COVID-19 in the New York City area," Journal of the American Medical Association, vol. 323, no. 20, pp. 2052-2059, 2020.

[16] R. Chen, W. Liang, M. Jiang et al., "Risk factors of fatal outcome in hospitalized subjects with coronavirus disease 2019 from a nationwide analysis in China," Chest, vol. 158, no. 1, pp. 97-105, 2020.

[17] X.-Y. Zhao, X.-X. Xu, H.-S. Yin et al., "Clinical characteristics of patients with 2019 coronavirus disease in a non-Wuhan area of Hubei Province, China: a retrospective study," $B M C$ Infectious Diseases, vol. 20, no. 1, p. 311, 2020.
[18] K. Brzeźniakiewicz-Janus, M. D. Lancé, A. Tukiendorf et al., "Selected hematological biomarkers to predict acute mortality in emergency department patients. Recent Polish hospital statistics," Disease Markers, vol. 2020, Article ID 8874361, 10 pages, 2020.

[19] L. Yan and Z. D. Hu, "Red blood cell distribution width, neutrophil-to-lymphocyte ratio, and in- hospital mortality in dyspneic patients admitted to the emergency department," Disease Markers, vol. 2020, Article ID 8839506, 5 pages, 2020.

[20] J. Budzianowski, K. Pieszko, P. Burchardt, J. Rzeźniczak, and J. Hiczkiewicz, "The role of hematological indices in patients with acute coronary syndrome," Disease Markers, vol. 2017, Article ID 3041565, 9 pages, 2017.

[21] N. Li, H. Zhou, and Q. Tang, "Red blood cell distribution width: a novel predictive indicator for cardiovascular and cerebrovascular diseases," Disease Markers, vol. 2017, Article ID 7089493, 23 pages, 2017.

[22] A. P. Yang, J. P. Liu, W. Q. Tao, and H. M. Li, "The diagnostic and predictive role of NLR, d-NLR and PLR in COVID-19 patients," International Immunopharmacology, vol. 84, article 106504, 2020.

[23] S. Sun, X. Cai, H. Wang et al., "Abnormalities of peripheral blood system in patients with COVID-19 in Wenzhou, China," Clinica Chimica Acta, vol. 507, pp. 174-180, 2020

[24] F. Zeng, L. Li, J. Zeng et al., "Can we predict the severity of coronavirus disease 2019 with a routine blood test?," Polish Archives of Internal Medicine, vol. 130, no. 5, pp. 400-406, 2020.

[25] D. McGonagle, K. Sharif, A. O’Regan, and C. Bridgewood, "The role of cytokines including interleukin-6 in COVID-19 induced pneumonia and macrophage activation syndromelike disease," Autoimmunity Reviews, vol. 19, no. 6, article 102537, 2020.

[26] N. Tang, H. Bai, X. Chen, J. Gong, D. Li, and Z. Sun, “Anticoagulant treatment is associated with decreased mortality in severe coronavirus disease 2019 patients with coagulopathy," Journal of Thrombosis and Haemostasis, vol. 18, no. 5, pp. 1094-1099, 2020.

[27] B. Tomar, H. J. Anders, J. Desai, and S. R. Mulay, "Neutrophils and neutrophil extracellular traps drive necroinflammation in COVID-19," Cell, vol. 9, no. 6, article E1383, 2020.

[28] T. Németh, M. Sperandio, and A. Mócsai, "Neutrophils as emerging therapeutic targets," Nature Reviews. Drug Discovery, vol. 19, no. 4, pp. 253-275, 2020.

[29] G. Schönrich and M. J. Raftery, "Neutrophil extracellular traps go viral," Frontiers in Immunology, vol. 7, p. 366, 2016.

[30] G. Chen, W. G. Di Wu, Y. Cao et al., "Clinical and immunological features of severe and moderate coronavirus disease 2019," The Journal of Clinical Investigation, vol. 130, no. 5, pp. 2620 2629, 2020.

[31] J. Liu, S. Li, J. Liu et al., "Longitudinal characteristics of lymphocyte responses and cytokine profiles in the peripheral blood of SARS-CoV-2 infected patients," eBioMedicine, vol. 55, article 102763, 2020.

[32] K. D. Kim, J. Zhao, and S. Auh, “Adaptive immune cells temper initial innate responses," Nature Medicine, vol. 13, no. 10, pp. 1248-1252, 2007.

[33] S. Q. Jiang, Q. F. Huang, W. M. Xie, C. Lv, and X. Q. Quan, "The association between severe COVID-19 and low platelet count: evidence from 31 observational studies involving 7613 participants," British Journal of Haematology, vol. 190, 2020. 
[34] J. Yang, M. Yang, F. Xu et al., "Effects of oxygen-induced lung damage on megakaryocytopoiesis and platelet homeostasis in a rat model," Pediatric Research, vol. 54, no. 3, pp. 344-352, 2003.

[35] T. M. Alempijevic, M. S. Lalosevic, I. Dumic et al., "Diagnostic accuracy of platelet count and platelet indices in noninvasive assessment of fibrosis in nonalcoholic fatty liver disease patients," Canadian Journal of Gastroenterology \& Hepatology, vol. 2017, article 6070135, 5 pages, 2017.

[36] F. X. Zhang, Z. L. Li, Z. D. Zhang, and X. C. Ma, "Prognostic value of red blood cell distribution width for severe acute pancreatitis," World Journal of Gastroenterology, vol. 25, no. 32, pp. 4739-4748, 2019.

[37] X. S. Yu, Z. Q. Chen, Y. F. Hu et al., "Red blood cell distribution width is associated with mortality risk in patients with acute respiratory distress syndrome based on the Berlin definition: a propensity score matched cohort study," Heart Lung, vol. 49, pp. 641-645, 2020.

[38] B. Wang, H. Lu, Y. Gong, B. Ying, and B. Cheng, "The association between red blood cell distribution width and mortality in critically ill patients with acute kidney injury," BioMed Research International, vol. 2018, Article ID 9658216, 7 pages, 2018.

[39] J. Li, X. Yang, J. Ma, F. Gong, and Q. Chen, "Relationship of red blood cell distribution width with cancer mortality in hospital," BioMed Research International, vol. 2018, Article ID 8914617, 8 pages, 2018.

[40] J. Howell, D. J. Pinato, R. Ramaswami et al., "Integration of the cancer-related inflammatory response as a stratifying biomarker of survival in hepatocellular carcinoma treated with sorafenib," Oncotarget, vol. 8, no. 22, pp. 36161-36170, 2017.

[41] M. Bellan, D. Soddu, E. Zecca et al., "Association between red cell distribution width and response to methotrexate in rheumatoid arthritis," Reumatismo, vol. 72, no. 1, pp. 16-20, 2020.

[42] M. Bellan, A. Giubertoni, C. Piccinino et al., "Red cell distribution width and platelet count as biomarkers of pulmonary arterial hypertension in patients with connective tissue disorders," Disease Markers, vol. 2019, Article ID 4981982, 7 pages, 2019. 